\section{Tissue absence initiates regeneration through Follistatin-mediated inhibition of Activin signaling}

\author{
Michael A Gaviño ${ }^{\dagger}$, Danielle Wenemoser ${ }^{\dagger}$, Irving E Wang, Peter W Reddien* \\ Department of Biology, Howard Hughes Medical Institute, Whitehead Institute, \\ Massachusetts Institute of Technology, Cambridge, United States
}

*For correspondence: reddien@ wi.mit.edu

tThese authors contributed equally to this work

Competing interests: The authors declare that no competing interests exist.

Funding: See page 11

Received: 14 September 2012 Accepted: 07 August 2013 Published: 10 September 2013

Reviewing editor: Marianne $\mathrm{E}$ Bronner, California Institute of Technology, United States

(c) Copyright Gaviño et al. This article is distributed under the terms of the Creative Commons Attribution License, which permits unrestricted use and redistribution provided that the original author and source are credited.

\begin{abstract}
Regeneration is widespread, but mechanisms that activate regeneration remain mysterious. Planarians are capable of whole-body regeneration and mount distinct molecular responses to wounds that result in tissue absence and those that do not. A major question is how these distinct responses are activated. We describe a follistatin homolog (Smed-follistatin) required for planarian regeneration. Smed-follistatin inhibition blocks responses to tissue absence but does not prevent normal tissue turnover. Two activin homologs (Smed-activin-1 and Smed-activin-2) are required for the Smed-follistatin phenotype. Finally, Smed-follistatin is wound-induced and expressed at higher levels following injuries that cause tissue absence. These data suggest that Smed-follistatin inhibits Smed-Activin proteins to trigger regeneration specifically following injuries involving tissue absence and identify a mechanism critical for regeneration initiation, a process important across the animal kingdom.

DOI: 10.7554/eLife.00247.001
\end{abstract}

\section{Introduction}

Regeneration occurs in widespread contexts and species. Invertebrates such as Hydra are capable of whole-animal regeneration from tissue fragments, and many vertebrates can regenerate appendages or repair damaged organs (Sánchez Alvarado, 2000). Despite this widespread relevance, the central mechanisms that drive regeneration are poorly understood.

Planarians are flatworms capable of regeneration following an almost limitless variety of injuries and have emerged as a powerful model for exploring the molecular underpinnings of regeneration (Newmark and Sánchez Alvarado, 2002). New tissues are formed at planarian wound sites in a process called blastema formation, and pre-existing tissues are reorganized after amputation to accommodate reduced animal size and further generate missing tissues (Morgan, 1901; Reddien and Sánchez Alvarado, 2004). The source of regenerated tissue in planarians is a population of adult dividing cells called neoblasts (Reddien and Sánchez Alvarado, 2004), which include pluripotent stem cells called clonogenic neoblasts (cNeoblasts) (Wagner et al., 2011). Neoblasts are the only somatic cycling cells in adult animals and can be specifically ablated by gamma irradiation, allowing for dissection of the requirements for neoblasts in regenerative processes (Reddien and Sánchez Alvarado, 2004). Recent work has described the earliest molecular and cellular events that occur following injury (Pellettieri et al., 2010; Wenemoser and Reddien, 2010; Sandmann et al., 2011; Wenemoser et al., 2012). One finding to emerge from this work is that animals initiate distinct cellular and molecular responses to 'major injuries' that remove significant amounts of tissue (e.g., head amputation) and to 'simple injuries' that require only minimal healing for repair (wounds that do not elicit blastema formation, such as punctures or incisions). Following simple injury, for example, animals display an increase in mitotic numbers $6 \mathrm{hr}$ after injury before returning to baseline levels (Wenemoser and Reddien, 2010), and expression of numerous wound-induced genes becomes undetectable by $24 \mathrm{hr}$ after injury (Wenemoser et al., 2012). Following a major injury, these same initial responses are 
eLife digest Most animals can respond to injury with some form of tissue regeneration. In mammals, this is limited to wound healing, whereas other vertebrates-such as salamanders and zebrafish - can regenerate parts of internal organs and even entire appendages. The planarian, a flatworm, is even more remarkable, being able to regenerate its head or tail following amputation, and even a whole animal from just a small body fragment. This is particularly impressive given that planarians have a complex internal anatomy, which includes muscles, intestines, a system similar to kidneys, and a central nervous system with a brain.

How is such regeneration accomplished? Why are planarians able to regenerate their bodies so extensively, whereas humans cannot? To what extent are the mechanisms of planarian regeneration common to other animals? These questions have driven the study of planarian regeneration for more than a century, but it is only in recent years that the tools needed to address these questions at the molecular level have become available.

Planarian regeneration proceeds over several days and involves multiple processes, including gene expression, cell division and cell death. Importantly, it has recently been shown that planarians activate different responses depending on whether an injury results in significant tissue loss-and therefore requires regeneration for repair-or if simple wound healing will be sufficient. The mechanisms behind these different responses to injury have, however, remained a mystery.

Now, Gaviño et al. have identified a key mechanism in the initiation of regeneration following tissue loss. This is centered on the gene follistatin, which is expressed following wounding. When genetic techniques are used to disrupt the expression of follistatin, regeneration is completely blocked. However, the animal's ability to routinely replace old cells via a stem-cell mediated mechanism is unaffected. This indicates that follistatin is specifically required for the replacement of cells lost through injury. Gaviño et al. further demonstrate that the protein encoded by follistatin likely initiates tissue regeneration upon substantial tissue loss through inhibition of proteins called Activins.

Activin and Follistatin proteins are broadly conserved in evolution, and are also expressed in mammals, raising the possibility that similar molecular circuits may govern regenerative responses in many species.

DOI: 10.7554/eLife.00247.002

observed, but subsequent responses are also activated: the $6 \mathrm{hr}$ increase in mitotic numbers is followed by a second increase $48 \mathrm{hr}$ after amputation (Wenemoser and Reddien, 2010), and woundinduced gene expression persists beyond $24 \mathrm{hr}$ and is refined over several days (Wenemoser et al., 2012). These responses are referred to as the 'missing-tissue response' (Wenemoser and Reddien, 2010; Wenemoser et al., 2012). How animals distinguish between injuries involving varying amounts of tissue loss and regulate these distinct wound response programs remains unknown.

We identified Smed-follistatin as required for molecular and cellular 'missing-tissue' responses during regeneration. Specifically, Follistatin-mediated inhibition of Activin signaling is required for regeneration to occur, with Smed-follistatin expression at wounds controlled by the extent of tissue absence following injury. These results suggest a mechanism by which regenerative responses can be initiated.

\section{Results}

\section{Smed-follistatin is a wound-induced gene required for regeneration}

To identify genes mediating regeneration-specific wound responses, we inhibited recently identified wound-induced genes (Wenemoser et al., 2012) with RNA interference (RNAi). Inhibition of Smedfollistatin (follistatin or fst), a gene encoding a Follistatin-like TGF- $\beta$-superfamily inhibitor, completely blocked regeneration (Figure 1A, Figure 1-figure supplement 1). No brain regeneration or anterior pole regeneration was observed in $f_{s t}(R N A i)$ animals (Figure 1A, Figure 1-figure supplement 2). The anterior pole phenotype is consistent with a described role for follistatin in anterior regeneration (Roberts-Galbraith and Newmark, 2013). fst(RNAi) animals, however, also failed to produce a blastema following either tail amputation or the excision of lateral tissue wedges that left anterior and posterior poles intact (Figure 1B). These data demonstrate that fst is required broadly for regeneration. 
A
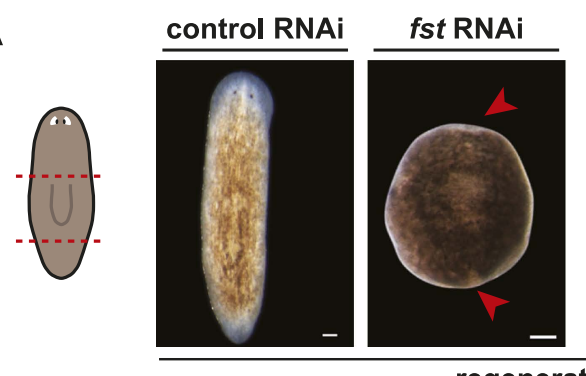

control RNAi

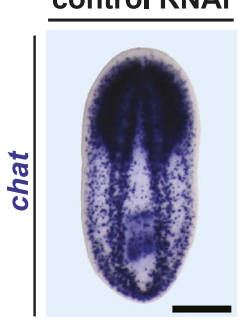

fst RNAi

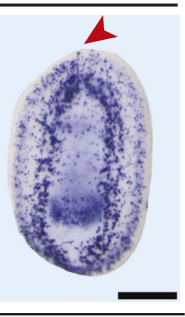

B

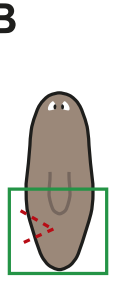

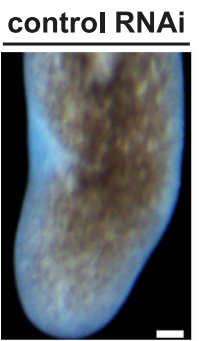

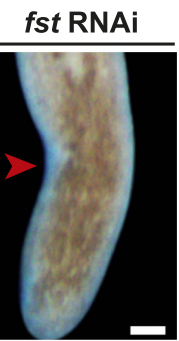

\section{C}

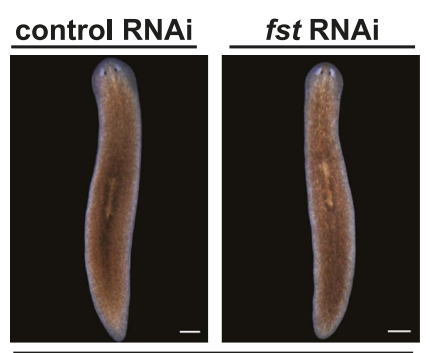

homeostasis $d>90$

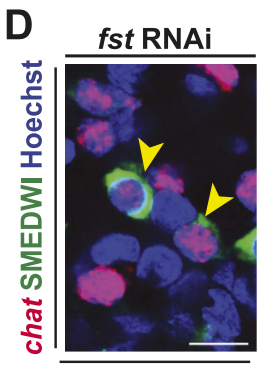

d20
E

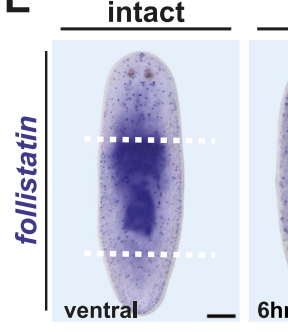

regeneration
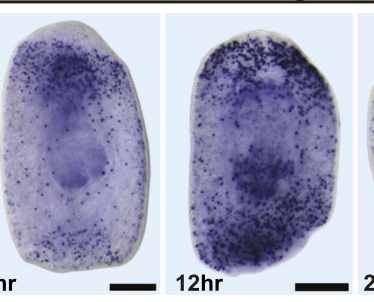

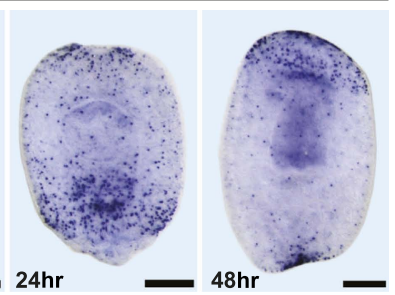

Figure 1. fst is wound induced and required for regeneration. (A) fst(RNAi) animals did not form blastemas after amputation (left, arrowheads, $n>100$ ) and did not regenerate a brain as assayed with an RNA probe for choline-acetyltransferase (chat, middle, arrowhead, $n=9 / 9)$. (B) fst(RNAi) animals did not form blastemas 8 days following excision of a wedge of lateral tissue (arrowhead, $n=14 / 14$ ). (C) fst(RNAi) animals displayed no phenotype in the absence of amputation (right, $n=30 / 30,123$ days RNAi). (D) fst(RNAi) animals still produced new neurons 20 days after failing to regenerate ( $\mathrm{n}=8 / 8$; cells that are SMEDWI protein ${ }^{+}$and chat ${ }^{+}$are newly differentiating neurons [Wagner et al., 2011]), demonstrating ongoing tissue turnover in old tissues. Scale bar $10 \mu \mathrm{m}$. (E) fst was expressed sparsely throughout the intact animal and at the anterior pole. Following head and tail amputation, robust fst expression occurred at both anterior and posterior wound sites, with peak expression $12 \mathrm{hr}$ post-amputation. Scale bars $=100 \mu \mathrm{m}$ for left of (A), (B), and (C); $200 \mu \mathrm{m}$ for right of (A) and (E). Anterior up.

DOI: 10.7554/eLife.00247.003

The following figure supplements are available for figure 1:

Figure supplement 1 . Specificity of the fst RNAi phenotype.

DOI: $10.7554 /$ eLife.00247.004

Figure supplement 2. Anterior pattern defects in fst(RNAi) animals.

DOI: $10.7554 /$ eLife.00247.005

Figure supplement 3. Efficacy of fst RNAi.

DOI: 10.7554/eLife.00247.006

Figure supplement 4. Wound-induced fst expression persists for several days after amputation.

DOI: 10.7554/eLife.00247.007

Figure supplement 5. fst is required after amputation for normal regeneration.

DOI: 10.7554/eLife.00247.008

Planarians constantly maintain adult tissues through cell turnover involving neoblasts (Reddien and Sánchez Alvarado, 2004). Consequently, most genes required for regeneration are also required for tissue turnover because of an involvement in neoblast biology (Reddien et al., 2005). Strikingly, unamputated fst(RNAi) animals did not shrink or lose structures, as is typically seen in animals with neoblast dysfunction, even after several months of significant expression reduction with RNAi (Figure 1C, Figure 1-figure supplement 3). Furthermore, amputated animals-despite failing to regeneratedisplayed ongoing long-term neoblast-based tissue turnover of remaining tissue (Figure 1D). Together, these data suggest that the requirement for fst in tissue replacement is specific to regeneration, as it is not detectably required for neoblast-mediated tissue turnover. Because of the rarity of genes required for regeneration but not tissue turnover, fst was a good candidate for specifically mediating the processes that occur following injury to bring about regeneration.

fst expression was induced at wounds by $6 \mathrm{hr}$ following amputation (Wenemoser et al., 2012; Roberts-Galbraith and Newmark, 2013) and persisted for several days, with maximal expression 
around $12 \mathrm{hr}$ post-amputation (Figure 1E, Figure 1-figure supplement 4). In unamputated animals, fst was expressed sparsely throughout the animal, including ventrally, in a thin peripheral domain, and at the anterior pole (Figure 1E, Figure 1-figure supplement 4; Roberts-Galbraith and Newmark, 2013). Injection of fst dsRNA only after amputation caused poor blastema formation and regeneration defects (Figure 1-figure supplement 5), consistent with a requirement for woundinduced fst expression in regeneration. We conclude that fst is a wound-induced factor required for regeneration.

\section{follistatin is required for the regeneration-specific neoblast response}

To characterize the defects underlying regeneration failure in fst(RNAi) animals, we first investigated whether fst regulates neoblast function in regeneration. Neoblasts can be visualized by detecting neoblast-specific transcripts through whole-mount in situ hybridization (Reddien et al., 2005) and quantified using flow cytometry (Hayashi et al., 2006). fst(RNAi) animals displayed normal neoblast numbers prior to amputation, indicating that the observed regeneration failure is not caused by neoblast loss (Figure 2A). We next assessed whether neoblasts respond to injury in fst(RNAi) animals. The neoblast response to injury involves two peaks (6 hr and $48 \mathrm{hr}$ post-amputation) in mitotic cell numbers, in between which neoblasts migrate to wounds (Wenemoser and Reddien, 2010). The first peak is generically induced by all injury types and is spatially widespread (Wenemoser and Reddien, 2010). The second peak occurs specifically following major injuries (removing tissues) and is biased toward wound sites (Wenemoser and Reddien, 2010). Amputated fst(RNAi) animals displayed a normal $6 \mathrm{hr}$ mitotic peak, indicating that a normal generic injury response was present (Figure 2B). By contrast, these animals failed to display a $48 \mathrm{hr}$ mitotic peak (Figure 2B). fst(RNAi) animals did however display localization of mitoses toward wound sites $48 \mathrm{hr}$ after amputation (Figure 2-figure supplement 1),

A

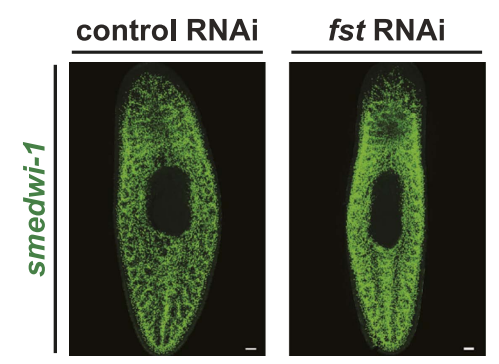

B

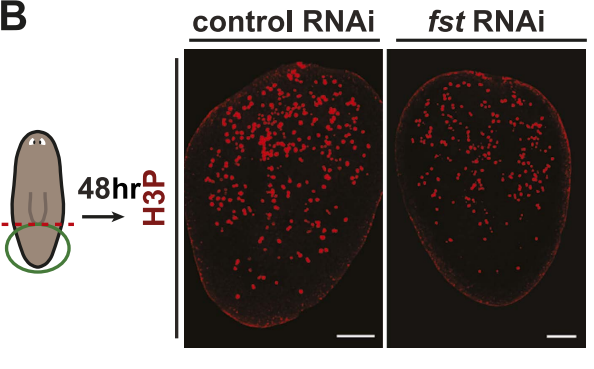

C

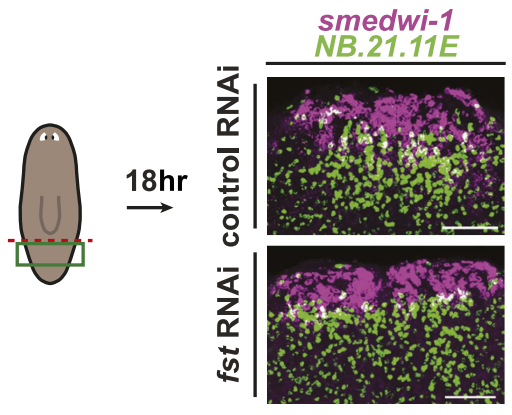

control RNAi fst RNAi
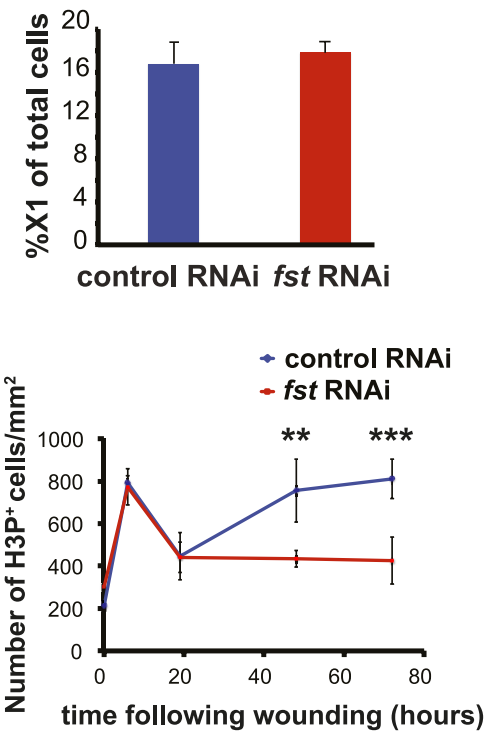

D

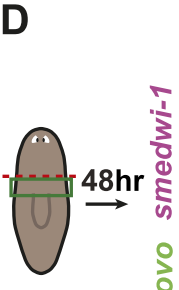

control RNAi

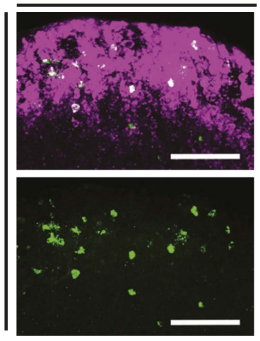

fst RNAi

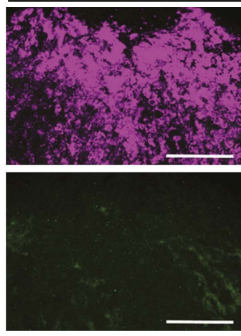

Figure 2. fst is required for the neoblast response to missing tissue. (A) fst RNAi did not affect neoblast number or distribution as assayed with an RNA probe for smedwi-1 $(n=5 / 5)$ and flow cytometry (percentage of live cells that were X1 cells). (B) fst(RNAi) tail fragments displayed reduced mitoses 48 hr and $72 \mathrm{hr}$ after amputation (right, $p<0.01$ and $p<0.001$, two-tailed t-test). (C) Neoblasts migrated to wounds in fst(RNAi) animals as assayed for the presence of smedwi-1+ cells at wounds (NB.21.11E+ cells mark pre-existing tissue). (D) fst(RNAi) animals lacked eye progenitors following head amputation as assayed with RNA probes for ovo $/$ smedwi-1+ $^{+}$cells $(p<0.001$, two-tailed $t$-test). Scale bars $=100 \mu m$. Anterior up. DOI: 10.7554/eLife.00247.009

The following figure supplements are available for figure 2:

Figure supplement 1. Neoblasts migrate to wounds normally in fst(RNAi) animals. DOI: $10.7554 /$ eLife.00247.010 
and neoblast enrichment at wound sites $18 \mathrm{hr}$ after injury (Figure 2C), indicating that neoblast migration occurred normally.

Given that fst(RNAi) animals displayed a defective proliferative response to missing tissue, we tested whether these animals produced regenerative progenitor cell types. Head amputation normally induces neoblasts to produce ovo ${ }^{+}$eye progenitors (Lapan and Reddien, 2012), but this process failed in fst(RNAi) animals (Figure 2D). From these data taken together, we conclude that fst is required for several aspects of the regeneration-specific neoblast response to injury.

\section{follistatin is required for responding to tissue absence following injury}

The abnormal missing-tissue-specific mitotic response of fst(RNAi) animals raised the possibility that other missing tissue responses could also require fst. Apoptosis increases following injury in planarians (Pellettieri et al., 2010), and, like the mitotic response, this increase involves a generic injury phase and a missing-tissue-specific phase. First, a local apoptosis burst occurs at wound sites 4 hr following any injury; second, a body-wide apoptosis burst occurs $72 \mathrm{hr}$ after injury, but only in cases involving missing tissue (Pellettieri et al., 2010). The apoptosis level in this latter phase scales with the amount of missing tissue (Pellettieri et al., 2010). Planarians possess a centrally located pharynx used for feeding and defecation (Reddien and Sánchez Alvarado, 2004); measuring apoptotic cell numbers by TUNEL within the pharynx is an established assay for quantifying the body-wide increase in apoptosis that occurs $72 \mathrm{hr}$ post-amputation (Pellettieri et al., 2010). Strikingly, fst(RNAi) pharynges displayed little increase in apoptotic cell numbers $72 \mathrm{hr}$ post amputation, whereas a roughly 20-fold increase from pre-amputation levels occurred in control pharynges (Figure 3A). fst(RNAi) animals had a normal $4 \mathrm{hr}$ apoptosis burst, indicating that fst is not generally required for apoptosis (Figure $3 B$ ). The $72 \mathrm{hr}$ apoptotic response occurs in animals that have had their neoblasts ablated and cannot regenerate (Pellettieri et al., 2010). Therefore, the failure of fst(RNAi) animals to produce this response cannot be explained as a non-specific result of regeneration failure.

In addition to the cellular responses to missing tissue described above, persistence of woundinduced gene expression is another aspect of the planarian missing-tissue response (Wenemoser et al., 2012). We observed less expression of two wound-response genes in fst(RNAi) animals than in controls 24-48 hr post-amputation, despite expression levels being indistinguishable at earlier timepoints (Figure 3C). Notably, some wound-induced genes display expression that inversely scales with missing tissue amount; for example, Smed-delta-1 displays higher expression after an incision or puncture (simple injuries) than after amputation (a major injury) (Wenemoser et al., 2012). Amputated fst(RNAi) animals displayed a higher, rather than lower, level of Smed-delta-1 expression than did controls $24 \mathrm{hr}$ after amputation (Figure 3D). Therefore, the lower expression levels observed for other wound-induced genes in fst(RNAi) animals do not reflect generically lower gene expression at wounds, but instead a specific requirement for fst for missing-tissue-specific gene expression.

Irradiated animals (which cannot regenerate) can display either higher or lower levels of woundinduced expression, depending on the gene examined (Wenemoser et al., 2012). Indeed, some wound-induced genes were similarly affected between irradiated and fst(RNAi) animals, while others were oppositely affected (Figure 3-figure supplement 1). As was the case for the failed apoptotic response of $f_{s t}(R N A i)$ animals, the missing-tissue gene expression defects of $f_{s t}(R N A i)$ animals cannot therefore be explained as a side-effect of regenerative failure.

In addition to producing a regeneration blastema, amputated animals must reorganize and rescale remaining tissue in a process termed morphallaxis (Morgan, 1901; Reddien and Sánchez Alvarado, 2004). Some aspects of this process do not require blastema formation. For example, wntP-2 (also known as wnt11-5 [Gurley et al., 2010]) is normally expressed in planarian tails (Petersen and Reddien, 2009; Gurley et al., 2010) and its expression domain restricts posteriorly within $48 \mathrm{hr}$ of amputation whether regeneration proceeds or not (Gurley et al., 2010). fst(RNAi) animals did not rescale the wntP-2 expression domain $48 \mathrm{hr}$ following amputation, further supporting a model in which fst is required for responding to missing tissue (Figure 3E). Following head amputation, head fragments not only produce posterior-specific cell types but also reduce numbers of anterior-specific cell types (which are overabundant for the new fragment dimensions). This process failed in fst(RNAi) head fragments (Figure 3F). Finally, fst(RNAi) fragments did not produce pharynges de novo (which normally occurs in pre-existing head and tail fragment tissue) (Figure 3-figure supplement 1). By contrast, RNAi of a different gene that blocked blastema formation (smad1) did not block pharynx formation, indicating 
A
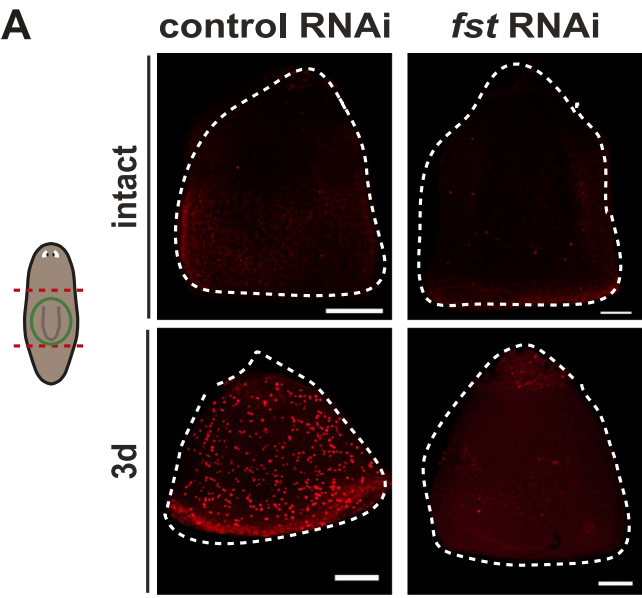

C

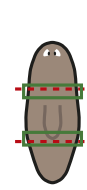

control RNAi fst RNAi
D

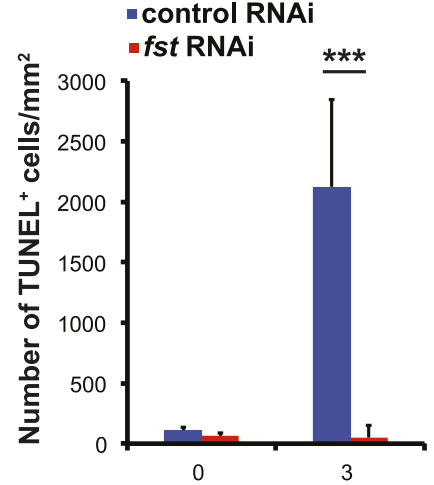

days following wounding
B

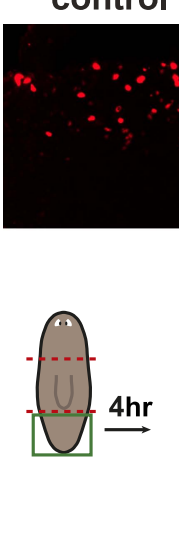

fst RNAi
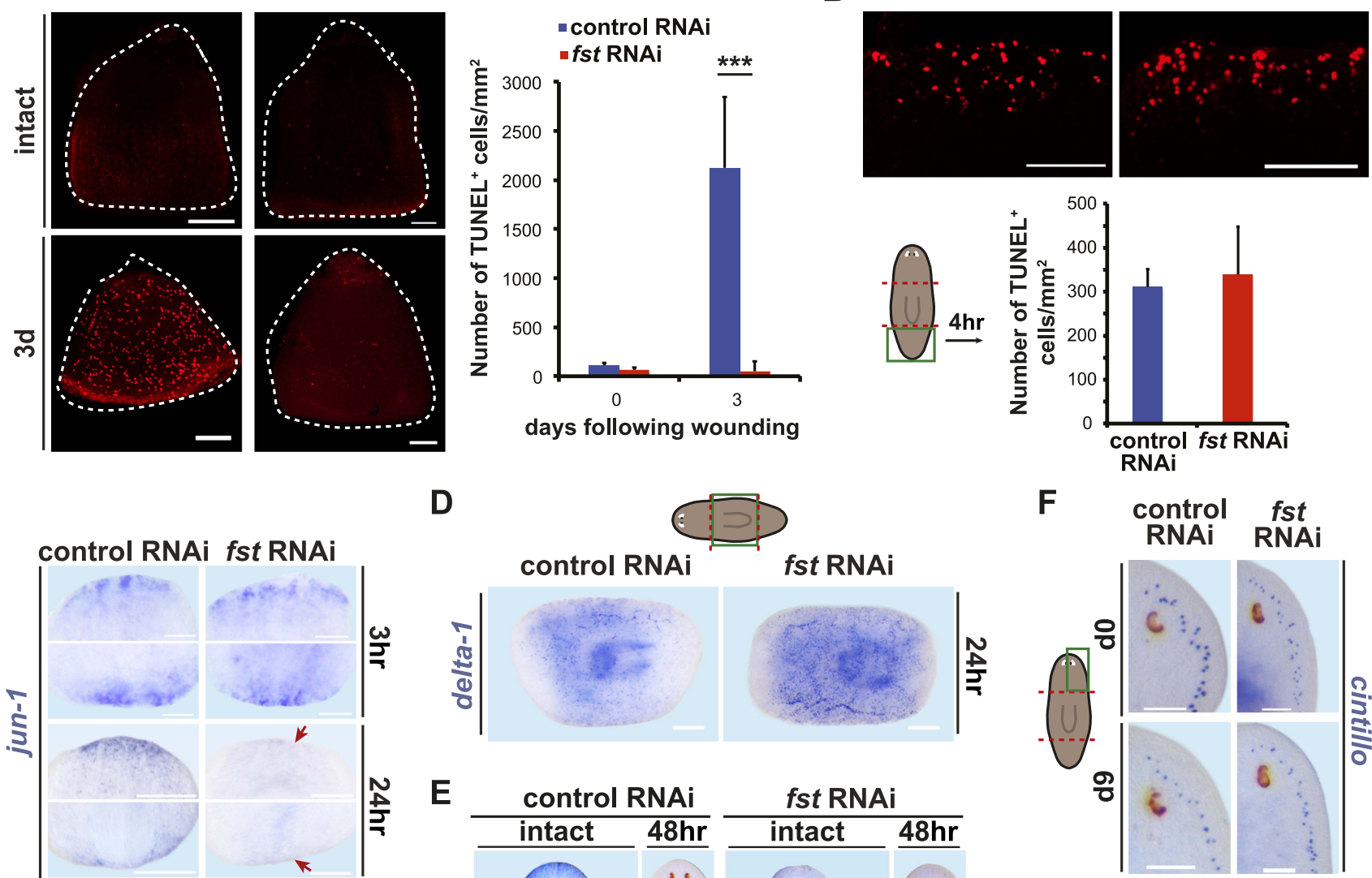

control RNAi

fst RNAi

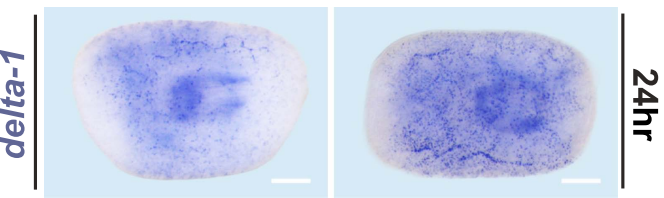

E

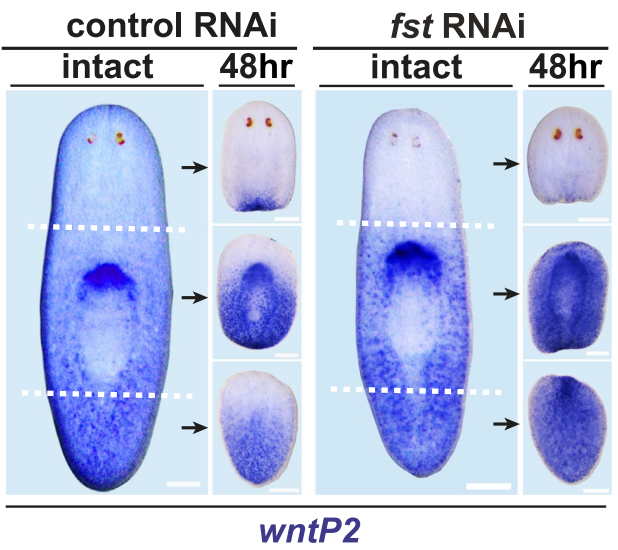

$\mathbf{F}$
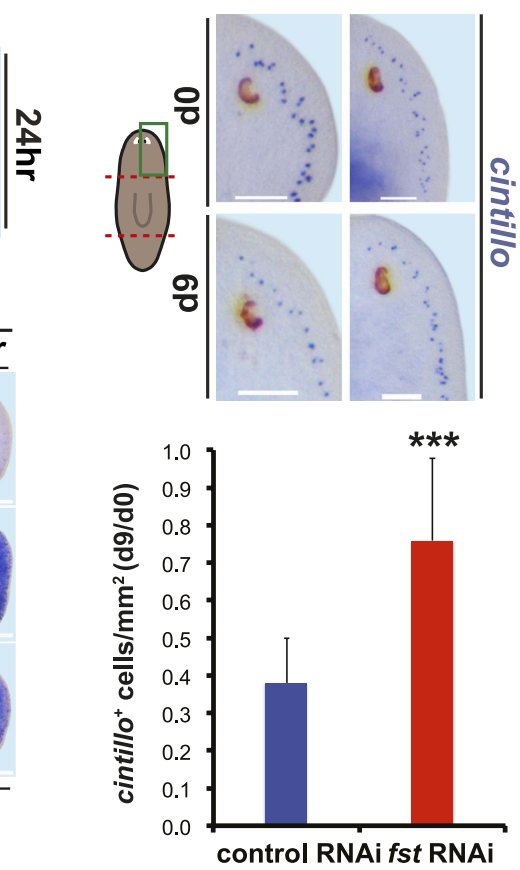

Figure 3. fst is required broadly for missing-tissue responses and morphallaxis. (A) fst(RNAi) animals displayed no increase in pharyngeal TUNEL ${ }^{+}$cells 3 days post-amputation ( $p<0.001$, two-tailed $t$-test). Dotted white line $=$ pharynx outline. (B) fst(RNAi) tail fragments displayed normal TUNEL ${ }^{+}$cell numbers $4 \mathrm{hr}$ post-amputation $(\mathrm{n}=6 / 6)$. (C) fst(RNAi) animals displayed normal wound-induced gene expression $3 \mathrm{hr}$ and $6 \mathrm{hr}$ after amputation (jun-1: $n=20 / 20, n \lg 1: n=5 / 5$ ) but reduced expression compared to controls $24-48 \mathrm{hr}$ after amputation (arrows; jun-1: 17/19 correctly scored blindly, p<0.01 Fisher's exact test, $n / g 1: 22 / 27$ correctly scored blindly, $p<0.01$, Fisher's exact test). (D) fst(RNAi) animals had increased wound-induced expression of delta-1 $24 \mathrm{hr}$ after amputation $(n=12 / 12)$. (E) fst(RNAi) animals did not rescale expression of wntP-2 $48 \mathrm{hr}$ after amputation ( $\mathrm{n}=18 / 21)$. (F) fst(RNAi) animals did not reduce the number of cintillo+ cells in head fragments following amputation $(p<0.001$, two-tailed t-test). Scale bars $=100 \mu m$. Anterior up in (A-C), (E), (F). Anterior left in (D).

DOI: 10.7554/eLife.00247.011

The following figure supplements are available for figure 3 :

Figure supplement 1. The fst(RNAi) phenotype is not a non-specific result of regeneration failure.

DOI: $10.7554 /$ eLife.00247.012

this defect is not a simple consequence of blastema formation failure (Figure 3-figure supplement 1). We conclude that $f_{s t}$ is required broadly for missing-tissue-specific wound responses, and that these defects likely underlie the inability of fst(RNAi) animals to regenerate. 


\section{Smed-activin-1 is required for the follistatin regeneration phenotype}

Because Follistatin proteins are well-characterized extracellular inhibitors of TGF- $\beta$ ligands (Nakamura et al., 1990; Hemmati-Brivanlou et al., 1994), we sought to identify putative TGF- $\beta$ ligands that Smed-Follistatin might regulate to promote regeneration. Seven putative TGF- $\beta$ superfamily members exist in the Schmidtea mediterranea genome (Figure 4-figure supplement 1 and Molina et al., 2007; Orii and Watanabe, 2007; Reddien et al., 2007; Gaviño and Reddien, 2011; Molina et al., 2011; Wenemoser et al., 2012; Roberts-Galbraith and Newmark, 2013). If Fst regulates one of the proteins encoded by these genes, then RNAi of that gene might suppress the fst RNAi phenotype. We tested this possibility (see Figure 4-figure supplement 2 and 'Materials and methods' for details) and found that RNAi of either of two genes, Smed-activin-1 (act-1 in short) or Smed-activin-2 (act-2), strongly suppressed the blastema formation defect (Figure 4A), the failure to regenerate a brain (Figure 4A), and the failed missing-tissue apoptotic response of fst(RNAi) animals (Figure 4B); RNAi of act-2 can also restore anterior pole regeneration in fst(RNAi) animals (Roberts-Galbraith and Newmark, 2013). Given that Follistatin proteins can directly regulate Activin proteins in other organisms (Nakamura et al., 1990; Hemmati-Brivanlou et al., 1994), these data suggest that Follistatin promotes missing tissue responses by inhibiting the function of Activin proteins.

\section{activin-1(RNAi) animals display excessive progenitor production following amputation}

Given that activin expression is required for the fst(RNAi) phenotype, we investigated the consequences of act-1 RNAi on regeneration. Although act-2(RNAi) has been reported to produce posterior regeneration defects (Roberts-Galbraith and Newmark, 2013), act-1(RNAi) animals were capable of regenerating (Figure 4-figure supplement 3, Figure 4-figure supplement 4) and, as with fst(RNAi), displayed normal neoblast turnover during homeostatic growth (Figure 4-figure supplement 5). act-1(RNAi) survived after amputation as well as controls did (observed more than a month following injury, $n=10 / 10$ ). act-1(RNAi) animals did however display some abnormalities. Although act-1(RNAi) animals displayed normal ovo ${ }^{+}$eye progenitor numbers prior to amputation, increased numbers as compared to controls were present following amputation (Figure 4C). By contrast, fst RNAi caused the opposite phenotype of reduced ovo ${ }^{+}$eye progenitor formation. These data raise the possibility that act-1 regulates responses to injury, with some aspects of regeneration overactive following act-1 inhibition.

\section{The amount of missing tissue regulates follistatin expression following injury}

Because $f s t$ is required for regeneration but not for normal tissue turnover, we reasoned that fst expression might be high following amputation, an injury type requiring significant tissue regeneration, but low following incision or puncture, injuries requiring only wound healing. We therefore assessed fst as compared to act expression at wounds following either incision or excision of a tissue wedge. Increased act-1 expression was not detected following either type of wound, with expression detected throughout the intestine of uninjured animals, suggesting an intestinal source of Activin-1 protein (Figure 5A). act-2 expression was similar to act-1 in intact animals, but unlike act-1 is woundinduced (Figure 5B, Figure 5-figure supplement 1; Roberts-Galbraith and Newmark, 2013). Indeed, act-2 was wound-induced following either incision or tissue wedge excision, with expression persisting for several days irrespective of injury severity (Figure 5C, Figure 5-figure supplement 2). By contrast, fst expression was induced at both wound types by $6 \mathrm{hr}$ after injury, but by $48 \mathrm{hr}$ after injury was present only at wedge excision wound sites (Figure 5C, Figure 5-figure supplement 2). These results indicate that fst expression persists longer at wounds that result in tissue absence. Furthermore, fst expression was greater at wounds involving a large amount of missing tissue (assessed at $48 \mathrm{hr}$ ) than at wounds with little missing tissue (Figure 5-figure supplement 3). Together, these data are consistent with a model in which wound-induced fst expression levels are regulated by the amount of missing tissue. In this model, fst promotes regenerative responses by inhibition of act- 1 and act-2 following major injury (Figure 5D).

\section{Discussion}

\section{Regeneration initiation}

All long-living animals face the prospect of injury and require regenerative mechanisms. Planarians are an exceptional example of the regenerative potential of animals. Distinct cellular and molecular 


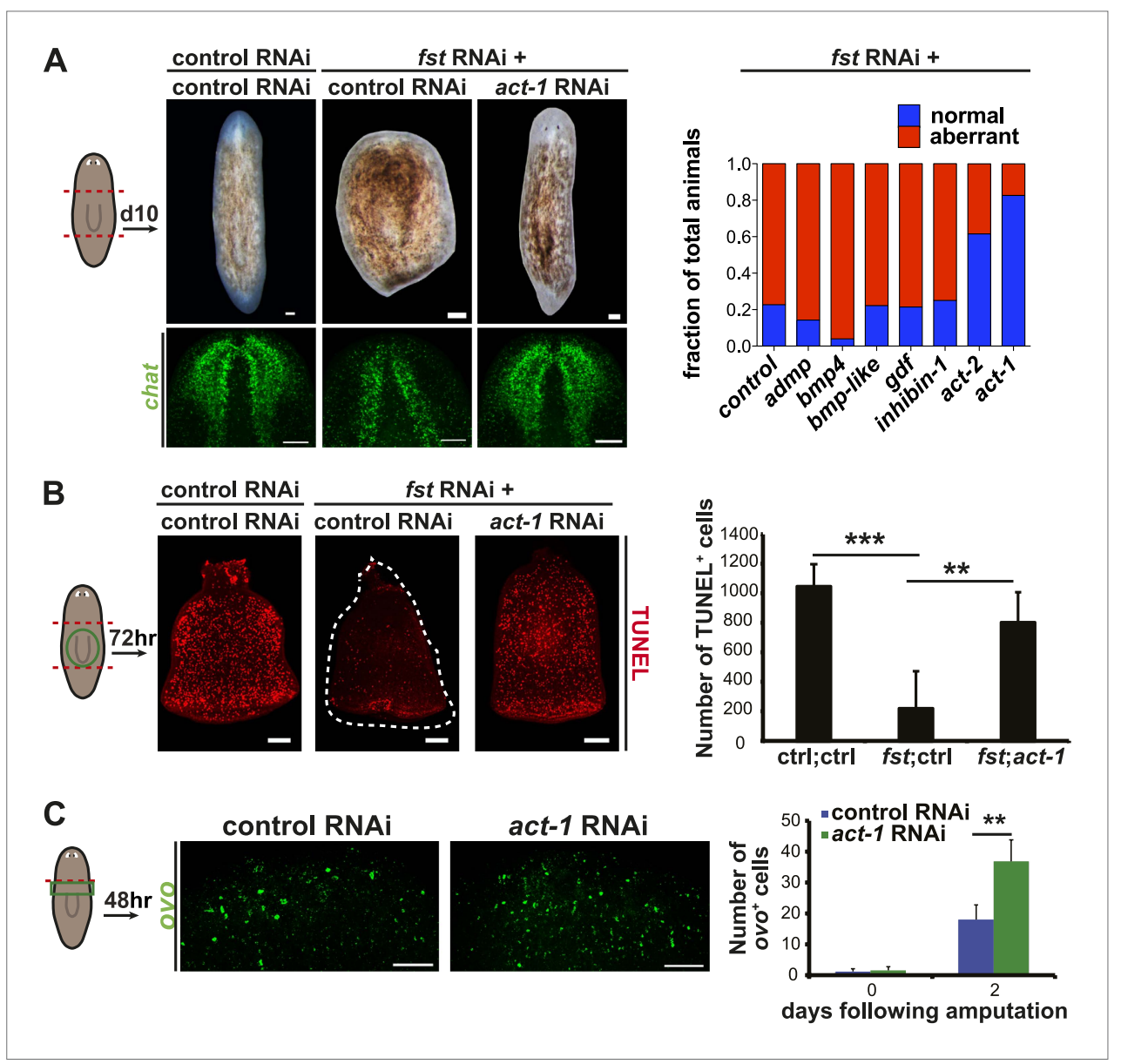

Figure 4. act-1 and act-2 are required for the fst RNAi phenotype. (A) fst(RNAi) animals treated with control dsRNA did not produce blastemas or a brain after amputation $(n=17 / 22)$, whereas fst(RNAi) animals treated with act-1 or act-2 dsRNA produced normal blastemas and brain (act-1: $n=19 / 23, p<0.0001$; act-2: $n=16 / 26, p<0.001$; Fisher's exact test for both). Inhibition of other candidate genes did not suppress the fst RNAi phenotype ( $>9$ for all other conditions). Aberrant animals were scored as having greatly decreased or absent brain. (B) fst(RNAi) animals treated with control dsRNA displayed a reduced apoptotic response 3 days after amputation, whereas fst(RNAi) animals treated with act- 1 dsRNA displayed a normal apoptotic response $(p<0.001$ between control RNAi and fst; ctrl RNAi; $p<0.01$ between fst; ctrl RNAi and fst;act-1 RNAi, two-tailed t-test for both). (C) act-1(RNAi) animals displayed a greater induction of ovo+ eye progenitors compared to controls 2 days after head amputation $(n=24$, $\mathrm{p}<0.0001$, two-tailed t-test). Scale bars $=100 \mu \mathrm{m}$. Anterior up.

DOI: 10.7554/eLife.00247.013

The following figure supplements are available for figure 4 :

Figure supplement 1. Phylogeny of planarian activin homologs.

DOI: 10.7554/eLife.00247.014

Figure supplement 2. RNAi controls for fst suppression experiments. DOI: 10.7554/eLife.00247.015

Figure supplement 3. Efficacy of act-1 RNAi. DOI: 10.7554/eLife.00247.016

Figure supplement 4. act-1(RNAi) animals appear normal following regeneration. DOI: 10.7554/eLife.00247.017

Figure supplement 5. act-1(RNAi) animals display normal neoblast numbers. DOI: 10.7554/eLife.00247.018

programs for responding to simple injury vs missing tissue exist in planarians. In the case of injuries involving substantial missing tissue, animals mount unique mitotic and apoptotic responses and produce an extended program of wound-induced gene expression (Pellettieri et al., 2010; Wenemoser 
A

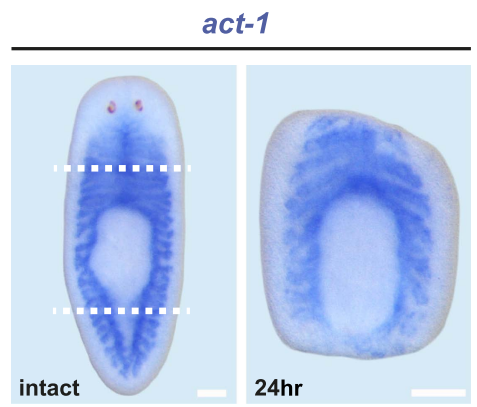

B

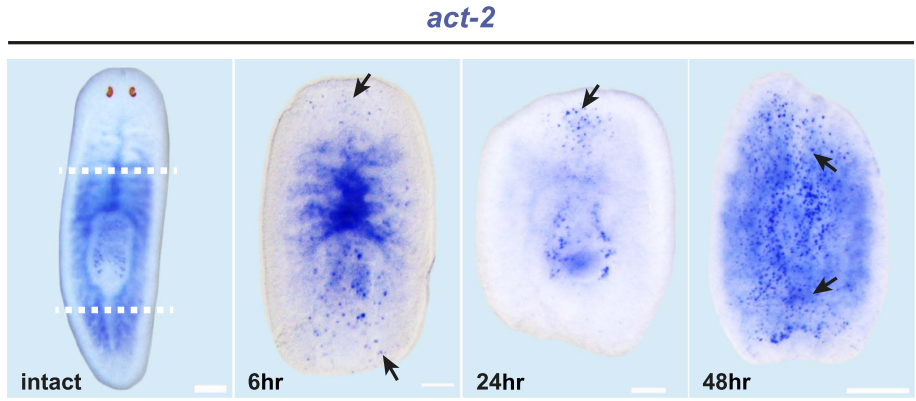

C
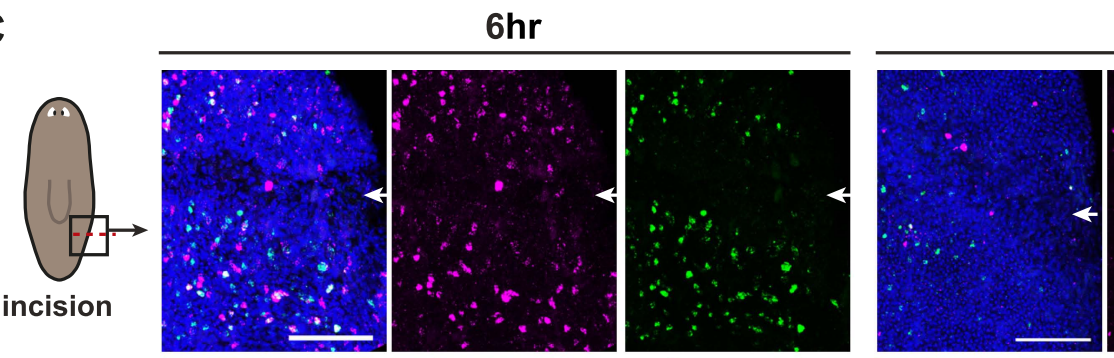

$48 \mathrm{hr}$
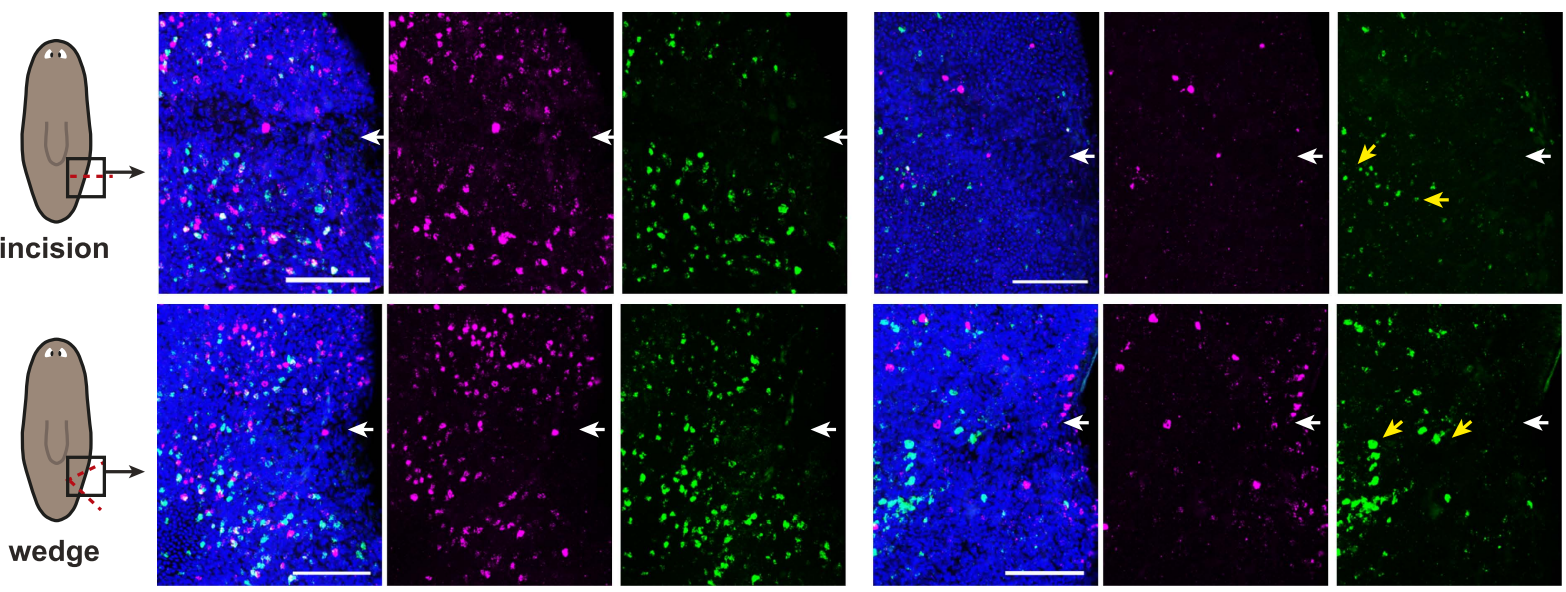

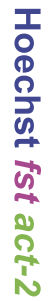

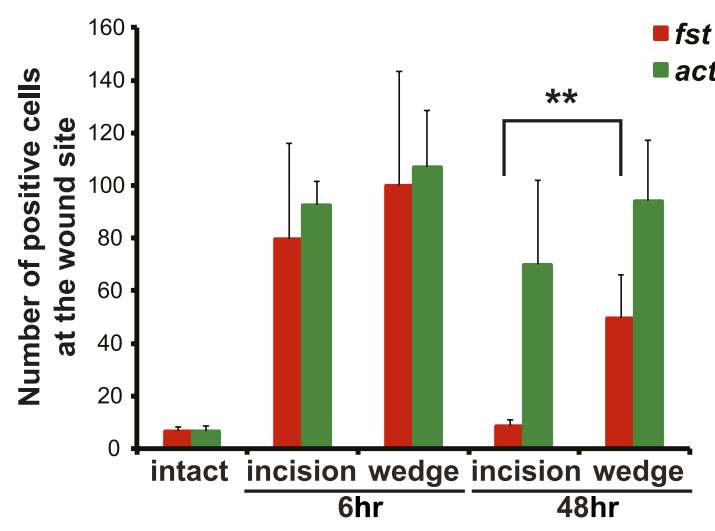

\section{Missing Tissue $\longrightarrow$ fst $^{\text {hi }} \longrightarrow$ act-1/2--Regenerative Responses}

No Missing Tissue $\longrightarrow \mathrm{fst}^{\mathrm{t}^{\circ}}{ }_{-}$act-1/2 $\longrightarrow$ Regenerative Responses

Figure 5. fst induction is regulated by tissue absence following injury. (A) act-1 was expressed broadly throughout the intestine and was not induced by amputation. (B) act-2 was expressed in the intestine and pharynx in intact animals and induced following amputation at wound sites $(6 \mathrm{hr}$ and $24 \mathrm{hr}$, arrows), eventually spreading throughout the body ( $48 \mathrm{hr}$, arrows). (C) Animals displayed wound-induced expression of fst $6 \mathrm{hr}$ after either incision or tissue wedge removal, but expression persisted only in cases of tissue wedge removal ( $n>5, p<0.01$, white arrowheads $=$ injury site). (D) A proposed genetic model for fst and act-1/2 in regeneration. Wounds induce fst expression. If there is missing tissue following injury, fst induction is high, Act-1/2 signaling is inhibited, and regeneration-specific responses are initiated. If there is no missing tissue following injury, fst expression is low, Act-1/2 signaling is not inhibited, and regeneration-specific responses are repressed. Scale bars $=100 \mu \mathrm{m}$. Anterior up.

DOI: 10.7554/eLife.00247.019

The following figure supplements are available for figure 5:

Figure supplement 1. Wound-induced act-2 expression persists for several days after amputation. DOI: 10.7554/eLife.00247.020

Figure supplement 2. fst and act-2 expression is negligible at wound sites prior to injury. DOI: 10.7554/eLife.00247.021

Figure supplement 3. The amount of missing tissue regulates wound-induced fst expression. DOI: 10.7554/eLife.00247.022 
and Reddien, 2010; Wenemoser et al., 2012). These events represent the earliest described divergent behaviors following major injuries requiring regeneration vs simple injuries requiring only wound healing. A central question has therefore become how these distinct responses are mediated.

We identified a gene encoding a homolog of the TGF- $\beta$ inhibitor, follistatin, that is required for regeneration and for regeneration-specific cellular and molecular responses to injury. Our data suggest that inhibition of Activin signaling by Fst is required for initiating a regenerative response at wounds following major injury. Finally, fst is wound-induced, with the level of fst expression persisting at high levels longer following a major injury than following a simple injury. We propose that wound-induced fst expression allows for regenerative responses to be initiated specifically as a consequence of tissue absence.

\section{The nature of the planarian missing-tissue response}

fst is the first gene known to be required for regeneration-specific responses in planarians. Not all missing-tissue responses are abolished following fst inhibition, however. For example, neoblast migration to amputation sites occurred normally in fst(RNAi) animals, despite the absence of a normal proliferative response. Similarly, although expression of act-1 and act-2 are required for the fst(RNAi) phenotype, inhibition of activin expression in the absence of amputation does not affect homeostatic tissue turnover or induce a regeneration-like state, demonstrating that the suppression of Activin alone is not sufficient to induce missing-tissue responses. Therefore, some aspects of the missingtissue response to injury require an as yet unknown 'missing-tissue' signal or signals that operate independently of $f s t$ and Activin signaling. Identifying and characterizing these processes will be important for understanding how the decision to mount a regenerative response occurs.

\section{TGF- $\beta$ signaling across regenerative contexts}

Our findings describe a system in which suppression of Activin signaling is required for regeneration. The possibility therefore exists that Activin signaling may serve similar functions in other organisms. Indeed, TGF- $\beta$ signaling has been implicated as a negative regulator of regeneration in a variety of contexts, including following partial hepatectomy (Russell et al., 1988; Kogure et al., 1995; RomeroGallo et al., 2005), in embryonic chick retinas (Sakami et al., 2008), in renal regeneration following ischemia/reperfusion injuries (Kojima et al., 2001), and for mouse skeletal muscle regeneration (Zhu et al., 2011). Given the relevance of these systems to human medicine, it will be important to investigate to what extent regenerative regimes recapitulate the mechanisms observed in planarians. Interestingly, a number of systems use TGF- $\beta$ signaling to promote rather than suppress regeneration: TGF- $\beta$ signaling is involved in axolotl limb and Xenopus tail regeneration (Lévesque et al., 2007; Ho and Whitman, 2008), activin expression can be induced by wounding and exogenous TGF- $\beta$ can speed healing in mammals (Mustoe et al., 1987; Hübner et al., 1996; Sulyok et al., 2004), TGF- $\beta$ signaling can promote regeneration following mouse ear hole-punching (Liu et al., 2011), and woundinduced activin promotes cell proliferation and migration following zebrafish fin amputation (Jaźwińska et al., 2007). Despite these contextual differences, TGF- $\beta$ signaling plays a major role in many forms of regeneration studied. Therefore, uncovering 'missing-tissue' signals in planarians, describing how these signals interact with Activin signaling, and identifying the key factors regulated by these signals will inform a broad understanding of core regenerative mechanisms.

\section{Materials and methods}

\section{Gene cloning}

For RNA probes, genes were cloned into pGEM and amplified with T7-promoter-containing primers. For RNAi, genes were cloned into pPR244 as described (Reddien et al., 2005). activin-1 was cloned with primers 5'-TCAACTGAAACGGAAGTTGG-3' and 5'-TGGTGGATCCTTACTTGCAG-3', activin-2 with primers 5'-ACCAATTATGGCCAATCCAG-3' and 5'-CCGGCTAATTGTGAACAAAC-3', and follistatin with 5'-CACAAGAGGCTGCAGTGAAT-3' and 5'-CATTCAGAAGGCATTGTCCA-3'.

\section{RNAi}

The control dsRNA for all RNAi experiments was unc-22 from Caenorhabditis elegans. RNAi experiments were performed by feeding a mixture of liver and bacteria expressing dsRNA (Reddien et al., 2005). $20 \mathrm{ml}$ of bacterial culture was pelleted and resuspended in $60 \mu$ l of liver. For fst and act- $1 \mathrm{RNAi}$ regeneration experiments, animals were fed on day 0 , day 4 , day 8 , and day 12 , amputated on day $16 / 17$ and either soaked for $6 \mathrm{hr}$ in $1 \mu \mathrm{g} / \mu \mathrm{l}$ dsRNA (TUNEL experiments), soaked for $2 \mathrm{hr}$ in dsRNA 
(gene expression experiments), or not soaked in dsRNA. For suppression experiments, totals from two separate experiments were pooled: (1) animals were fed fst dsRNA on day 0, day 4, day 8, and day 12, fed candidate gene dsRNA on day 16, day 20, and day 23, and amputated on day 24. (2) Animals were amputated and injected four times with a $30 \mathrm{nl}$ equimolar mixture of fst and candidate gene dsRNA on day 0 , injected without amputation on day 1 , amputated and injected on day 4, and injected only on day 5 . Animals were scored and fixed 8 days after the final amputation.

\section{In situ hybridizations, immunostaining, and TUNEL}

Whole-mount in situ hybridizations and fluorescence in situ hybridizations (FISH) were performed as described (Pearson et al., 2009). For double/triple labeling, HRP-inactivation was performed between labelings (4\% formaldehyde, $30 \mathrm{~min}$ ). Immunostainings were performed as previously described (Reddien et al., 2005) using tyramide signal enhancement. TUNEL was performed as previously described (Pellettieri et al., 2010).

\section{Y-irradiation}

For elimination of neoblasts, planarians were exposed to $6000 \mathrm{rad}(6 \mathrm{~K}, \sim 72 \mathrm{~min})$ using a cesium source ( 83 $\mathrm{rad} / \mathrm{min})$.

\section{Flow cytometry}

Animals were amputated in cold CMFB, and cells prepared as described (Scimone et al., 2011). For quantification of $\mathrm{X} 1$ cells, five animals were used per RNAi condition in triplicate. Analyses and sorting were performed using a Moflo3 FACS sorter (Dako-Cytomation, Carpinteria, CA) and FlowJo.

\section{Imaging and analyses}

For quantifying cell numbers expressing a marker or an area of positive cells, equal numbers of optical stacks were taken per specimen, collapsed, and quantified using Automeasure in AxioVision (Zeiss, Jena, Germany) or manually. For quantification of fluorescence intensity, 7 optical stacks were acquired from the ventral surface of animals, collapsed, and values determined using the Automeasure module (Densitometric sum) in AxioVision (Zeiss). Images were acquired using an Axiolmager with Apotome (Zeiss) or an LSM 700 (Zeiss).

\section{Acknowledgements}

We thank Reddien Lab members for comments and discussion. PWR is an early career scientist of the Howard Hughes Medical Institute and an associate member of the Broad Institute of Harvard and MIT. We acknowledge support from the NIH (R01GM080639) and the Keck Foundation.

\section{Additional information}

Funding

\begin{tabular}{lll} 
Funder & $\begin{array}{l}\text { Grant reference } \\
\text { number }\end{array}$ & Author \\
\hline National Institutes of Health & R01GM080639 & Peter W Reddien \\
\hline Howard Hughes Medical Institute & & Peter W Reddien \\
\hline Keck Foundation & & Peter W Reddien
\end{tabular}

The funders had no role in study design, data collection and interpretation, or the decision to submit the work for publication.

Author contributions

MAG, DW, IEW, Conception and design, Acquisition of data, Analysis and interpretation of data, Drafting or revising the article; PWR, Conception and design, Analysis and interpretation of data, Drafting or revising the article

\section{References}

Castresana J. 2000. Selection of conserved blocks from multiple alignments for their use in phylogenetic analysis. Mol Biol Evol 17:540-552. doi: 10.1093/oxfordjournals.molbev.a026334. 
Eisenhoffer GT, Kang H, Sánchez Alvarado A. 2008. Molecular analysis of stem cells and their descendants during cell turnover and regeneration in the planarian Schmidtea mediterranea. Cell Stem Cell 3:327-39. doi: 10.1016/j.stem.2008.07.002.

Gaviño MA, Reddien PW. 2011. A Bmp/Admp regulatory circuit controls maintenance and regeneration of dorsal-ventral polarity in planarians. Curr Biol 21:294-9. doi: 10.1016/j.cub.2011.01.017.

Gurley KA, Elliott SA, Simakov O, Schmidt HA, Holstein TW, Sánchez Alvarado A. 2010. Expression of secreted Wnt pathway components reveals unexpected complexity of the planarian amputation response. Dev Biol 347:24-39. doi: 10.1016/j.ydbio.2010.08.007.

Hayashi T, Asami M, Higuchi S, Shibata N, Agata K. 2006. Isolation of planarian X-ray-sensitive stem cells by fluorescence-activated cell sorting. Dev Growth Differ 48:371-80. doi: 10.1111/j.1440-169X.2006.00876.x.

Hemmati-Brivanlou A, Kelly OG, Melton DA. 1994. Follistatin, an antagonist of activin, is expressed in the Spemann organizer and displays direct neuralizing activity. Cell 77:283-95. doi: 10.1016/0092-8674(94)90320-4.

Higgins DG. 1994. CLUSTAL V: multiple alignment of DNA and protein sequences. Methods Mol Biol 25:307-18. doi: 10.1385/0-89603-276-0:307.

Ho DM, Whitman M. 2008. TGF-ß signaling is required for multiple processes during Xenopus tail regeneration. Dev Biol 315:203-16. doi: 10.1016/j.ydbio.2007.12.031.

Hübner G, Hu Q, Smola H, Werner S. 1996. Strong induction of activin expression after injury suggests an important role of activin in wound repair. Dev Biol 173:490-8. doi: 10.1006/dbio.1996.0042.

Huelsenbeck JP, Ronquist F. 2001. MRBAYES: Bayesian inference of phylogenetic trees. Bioinformatics 17:754-5. doi: 10.1093/bioinformatics/17.8.754.

Jaźwińska A, Badakov R, Keating MT. 2007. Activin-ßA signaling is required for zebrafish fin regeneration. Curr Biol 17:1390-5. doi: 10.1016/j.cub.2007.07.019.

Kogure K, Omata W, Kanzaki M, Zhang YQ, Yasuda H, Mine T, et al. 1995. A single intraportal administration of follistatin accelerates liver regeneration in partially hepatectomized rats. Gastroenterology 108:1136-42. doi: 10.1016/0016-5085(95)90212-0.

Kojima I, Maeshima A, Zhang YQ. 2001. Role of the activin-follistatin system in the morphogenesis and regeneration of the renal tubules. Mol Cell Endocrinol 180:179-82. doi: 10.1016/S0303-7207(01)00511-1.

Lapan SW, Reddien PW. 2012. Transcriptome analysis of the planarian eye identifies ovo as a specific regulator of eye regeneration. Cell Rep 2:294-307. doi: 10.1016/j.celrep.2012.06.018.

Lévesque M, Gatien S, Finnson K, Desmeules S, Villiard E, Pilote M, et al. 2007. Transforming growth factor: B signaling is essential for limb regeneration in axolotls. PLOS ONE 2:e1227. doi: 10.1371/journal.pone.0001227.

Liu J, Johnson K, Li J, Piamonte V, Steffy BM, Hsieh MH, et al. 2011. Regenerative phenotype in mice with a point mutation in transforming growth factor $ß$ type I receptor (TGFBR1). Proc Natl Acad Sci USA 108:14560-5. doi: 10.1073/pnas.1111056108.

Molina MD, Neto A, Maeso I, Gómez-Skarmeta JL, Saló E, Cebrià F. 2011. Noggin and noggin-like genes control dorsoventral axis regeneration in planarians. Curr Biol 21:300-5. doi: 10.1016/j.cub.2011.01.016.

Molina MD, Saló E, Cebrià F. 2007. The BMP pathway is essential for re-specification and maintenance of the dorsoventral axis in regenerating and intact planarians. Dev Biol 311:79-94. doi: 10.1016/j.ydbio.2007.08.019.

Morgan TH. 1901. Regeneration. New York: Macmillan.

Mustoe TA, Pierce GF, Thomason A, Gramates P, Sporn MB, Deuel TF. 1987. Accelerated healing of incisional wounds in rats induced by transforming growth factor-beta. Science 237:1333-6. doi: 10.1126/science.2442813.

Nakamura T, Takio K, Eto Y, Shibai H, Titani K, Sugino H. 1990. Activin-binding protein from rat ovary is follistatin. Science 247:836-8. doi: 10.1126/science.2106159.

Newmark PA, Sánchez Alvarado A. 2002. Not your father's planarian: a classic model enters the era of functional genomics. Nat Rev Genet 3:210-9. doi: 10.1038/nrg759.

Orii H, Watanabe K. 2007. Bone morphogenetic protein is required for dorso-ventral patterning in the planarian Dugesia japonica. Dev Growth Differ 49:345-9. doi: 10.1111/j.1440-169X.2007.00931.x.

Pearson BJ, Eisenhoffer GT, Gurley KA, Rink JC, Miller DE, Sánchez Alvarado A. 2009. Formaldehyde-based whole-mount in situ hybridization method for planarians. Dev Dyn 238:443-50. doi: 10.1002/dvdy.21849.

Pellettieri J, Fitzgerald P, Watanabe S, Mancuso J, Green DR, Sánchez Alvarado A. 2010. Cell death and tissue remodeling in planarian regeneration. Dev Bio/ 338:76-85. doi: 10.1016/j.ydbio.2009.09.015.

Petersen CP, Reddien PW. 2009. A wound-induced Wnt expression program controls planarian regeneration polarity. Proc Natl Acad Sci USA 106:17061-6. doi: 10.1073/pnas.0906823106.

Reddien PW, Bermange AL, Kicza AM, Sánchez Alvarado A. 2007. BMP signaling regulates the dorsal planarian midline and is needed for asymmetric regeneration. Development 134:4043-51. doi: 10.1242/dev.007138.

Reddien PW, Bermange AL, Murfitt KJ, Jennings JR, Sánchez Alvarado A. 2005. Identification of genes needed for regeneration, stem cell function, and tissue homeostasis by systematic gene perturbation in planaria. Dev Cell 8:635-49. doi: 10.1016/j.devcel.2005.02.014.

Reddien PW, Oviedo NJ, Jennings JR, Jenkin JC, Sánchez Alvarado A. 2005. SMEDWI-2 is a PIWI-like protein that regulates planarian stem cells. Science 310:1327-30. doi: 10.1126/science.1116110.

Reddien PW, Sánchez Alvarado A. 2004. Fundamentals of planarian regeneration. Annu Rev Cell Dev Biol 20:725-57. doi: 10.1146/annurev.cellbio.20.010403.095114.

Roberts-Galbraith RH, Newmark PA. 2013. Follistatin antagonizes activin signaling and acts with Notum to direct planarian head regeneration. Proc Natl Acad Sci USA 110:1363-8. doi: 10.1073/pnas.1214053110.

Romero-Gallo J, Sozmen EG, Chytil A, Russell WE, Whitehead R, Parks WT, et al. 2005. Inactivation of TGF- $\beta$ signaling in hepatocytes results in an increased proliferative response after partial hepatectomy. Oncogene 24:3028-41. doi: 10.1038/sj.onc.1208475. 
Ronquist F, Huelsenbeck JP. 2003. MrBayes 3: Bayesian phylogenetic inference under mixed models. Bioinformatics 19:1572-4. doi: 10.1093/bioinformatics/btg180.

Russell WE, Coffey RJ Jr, Ouellette AJ, Moses HL. 1988. Type beta transforming growth factor reversibly inhibits the early proliferative response to partial hepatectomy in the rat. Proc Natl Acad Sci USA 85:5126-30. doi: 10.1073/pnas.85.14.5126.

Sakami S, Etter P, Reh TA. 2008. Activin signaling limits the competence for retinal regeneration from the pigmented epithelium. Mech Dev 125:106-16. doi: 10.1016/j.mod.2007.10.001.

Sánchez Alvarado A. 2000. Regeneration in the metazoans: why does it happen? Bioessays 22:578-90. doi: 10.1002/(SICI)1521-1878(200006)22:6<578::AID-BIES11>3.0.CO;2-\#.

Sandmann T, Vogg MC, Owlarn S, Boutros M, Bartscherer K. 2011. The head-regeneration transcriptome of the planarian Schmidtea mediterranea. Genome Biol 12:R76. doi: 10.1186/gb-2011-12-8-r76.

Scimone ML, Srivastava M, Bell GW, Reddien PW. 2011. A regulatory program for excretory system regeneration in planarians. Development 138:4387-98. doi: 10.1242/dev.068098.

Sulyok S, Wankell M, Alzheimer C, Werner S. 2004. Activin: an important regulator of wound repair, fibrosis, and neuroprotection. Mol Cell Endocrinol 225:127-32. doi: 10.1016/j.mce.2004.07.011.

Thompson JD, Higgins DG, Gibson TJ. 1994. CLUSTAL W: improving the sensitivity of progressive multiple sequence alignment through sequence weighting, position-specific gap penalties and weight matrix choice. Nucleic Acids Res 22:4673-80. doi: 10.1093/nar/22.22.4673.

Wagner DE, Wang IE, Reddien PW. 2011. Clonogenic neoblasts are pluripotent adult stem cells that underlie planarian regeneration. Science 332:811-6. doi: 10.1126/science.1203983.

Wenemoser D, Lapan SW, Wilkinson AW, Bell GW, Reddien PW. 2012. A molecular wound response program associated with regeneration initiation in planarians. Genes Dev 26:988-1002. doi: 10.1101/gad.187377.112.

Wenemoser D, Reddien PW. 2010. Planarian regeneration involves distinct stem cell responses to wounds and tissue absence. Dev Biol 344:979-91. doi: 10.1016/j.ydbio.2010.06.017.

Zhu J, Li Y, Lu A, Gharaibeh B, Ma J, Kobayashi T, et al. 2011. Follistatin improves skeletal muscle healing after injury and disease through an interaction with muscle regeneration, angiogenesis, and fibrosis. Am J Pathol 179:915-30. doi: 10.1016/j.ajpath.2011.04.008. 